\title{
Low-molecular-weight heparins as adjunctive therapy to thrombolytics: extracting the best data
}

\author{
Alison L. Bailey, Steven R. Steinhubl
}

$\infty$

See related article page I42I

I $\mathrm{n}$ the United States, ST-segment elevation myocardial infarction (STEMI) affects about 500 ooo people annually; in Canada, about 50 ooo. The time to restoration of normal blood flow in the occluded artery is accepted to be a key determinant of short- and long-term outcomes, whether reperfusion is accomplished by thrombolysis or percutaneous coronary intervention (PCI). The main limitation of thrombolysis, however, has always been its unreliability in restoring coronary flow: restoration is complete in only somewhat more than half of the patients so treated.

In this issue of CMAJ, Armstrong and colleagues ${ }^{1}$ have evaluated the safety and efficacy of the thrombolytic drug tenecteplase plus either enoxaparin (a low-molecular-weight heparin $[\mathrm{LMWH}]$ ) or unfractionated heparin in cases of STEMI by doing a pooled analysis of data from the ASSENT (Assessment of the Safety and Efficacy of a New Thrombolytic Regimen)-3 and ASSENT- 3 PLUS trials. The ASSENT- 3 trial was designed to evaluate tenecteplase administered in hospital along with enoxaparin, abciximab or unfractionated heparin to patients after STEMI. The investigators ${ }^{2}$ concluded that the frequency of ischemic complications of acute myocardial infarction was lower with tenecteplase plus enoxaparin or abciximab than with tenecteplase plus unfractionated heparin. The ASSENT3 PLUS trial, an extension of that study, was designed to evaluate tenecteplase with either enoxaparin or unfractionated heparin in patients after STEMI, but in the prehospital setting. Although the combination of tenecteplase and enoxaparin reduced the incidence of ischemic complications of acute myocardial infarction, it was also found to be associated with an increased risk of major bleeding and intracranial hemorrhage among patients 75 years of age and older. ${ }^{3}$ For the current analysis, data from the patients who received tenecteplase plus enoxaparin $(n=2040)$ and tenecteplase plus unfractionated heparin $(n=2038)$ in the ASSENT- 3 trial were pooled with those from patients in the ASSENT- 3 PLUS trial $(n=8 \mathrm{r} 8)$.

Armstrong's group arrived at several conclusions based on the pooled data. First, the reduction in rates of reinfarction and recurrent ischemia was greater among patients treated with tenecteplase plus enoxaparin than among those given tenecteplase plus unfractionated heparin, but with a com$\infty$ mensurate excess in major systemic bleeding. These results are akin to those seen in several other studies that compared LMWH with unfractionated heparin in the setting of thrombolysis. A recent meta-analysis ${ }^{4}$ that compared the effects of these anticoagulants in people given thrombolytics after STEMI reviewed 6 trials involving 7098 patients in total. A decreased rate of reinfarction and an increased incidence of mi- nor bleeding, along with a nonsignificant increase in stroke, was associated with LMWH compared with unfractionated heparin. In contrast, in a recent substudy ${ }^{5}$ from the CLARITYTIMI (Clopidogrel as Adjunctive Reperfusion TherapyThrombolysis in Myocardial Infarction) 28 trial, which involved patients younger than 75 years who were given thrombolytics and randomly assigned to receive either clopidogrel or a placebo, no increase in bleeding complications or stroke was found in the LMWH group. Angiographic results were improved and rates of adverse cardiovascular events were lower than in the group provided unfractionated heparin. ${ }^{5}$

\section{Thrombolysis alone re- stores coronary flow com- pletely in only about half of patients so treated.}

Second, the patients in the enoxaparin group who underwent urgent revascularization seemed to derive greater protection from reinfarction. Again, supporting evidence was found in the CLARITY-TIMI 28 substudy. Patients receiving LMWH had a lower combined rate of an infarct-related occluded artery or death, or recurrent myocardial infarction before angiography than those receiving unfractionated heparin. ${ }^{5}$ Moreover, in TIMI no excess major bleeding or intracranial hemorrhage was observed, even when LMWH was combined with clopidogrel, given in the setting of angiography or PCI, used in conjunction with glycoprotein IIb or IIIa, or administered to patients with moderately impaired renal function. Although these results are interesting, some issues remain unresolved. No information was provided about the anticoagulation regimen used for revascularization. Was enoxaparin continued, or were some crossover patients reassigned to receive unfractionated heparin? Obviously, this would have important implications about how best to interpret these results in light of the findings of the SYNERGY (Superior Yield of the New Strategy of Enoxaparin, Revascularization and Glycoprotein IIb/IIIa Inhibitors) trial. ${ }^{6}$

Third, the excess incidence of major systemic bleeding seen with enoxaparin was observed in all groups given that form of heparin, but it was more prominent among the pa- 
tients who did not undergo revascularization. The reason for this is not entirely clear. It may be a reflection of the shorter duration of the treatment and the higher incidence of subtherapeutic anticoagulation achieved with unfractionated heparin. On the other hand, it may simply reflect the fact that patients who went on to have nonurgent catheterization and revascularization were exposed to shorter periods of anticoagulation and therefore were at lower risk of bleeding.

Finally, one of the most important observations from the ASSENT-3 PLUS trial (which was not seen in the ASSENT-3 trial) was an increased risk of intracranial hemorrhage among patients (especially women) older than 75 years who received enoxaparin and tenecteplase. Although of concern, this finding is problematic for several reasons. The proportion of elderly women in the ASSENT-3 PLUS trial was larger in the enoxaparin group than in the unfractionated heparin group ( $38 \%$ v. $30 \%$ ), and about $20 \%$ of all the ASSENT- 3 PLUS patients received more than $105 \%$ of the correct dose of tenecteplase. ${ }^{3}$ Only $6 \mathrm{I} \%$ of the patients in the unfractionated heparin group received an intravenous (IV) bolus and drip of unfractionated heparin before arrival at the hospital, and only $30 \%$ were within the target range of their activated partial thromboplastin time (i.e., the preferred aPTT range) during their 48 hours of unfractionated heparin administration, whereas $88 \%$ of the patients in the enoxaparin group received their IV bolus and subcutaneous dose of enoxaparin on schedule. In addition, nonscheduled LMWH was given to $33 \%$ of the patients in the enoxaparin group and $47 \%$ of those in the unfractionated heparin group during their hospital stay. It has been known for years that the risk of intracranial hemorrhage decreases with the dose of heparin; ${ }^{7}$ it is therefore not surprising that those in the unfractionated heparin group had lower rates in this study. The number of patients who received a therapeutic dose of unfractionated heparin was low at best, whereas the number receiving the target dose of enoxaparin was much higher. Although all these confounding variables factor into the need to further evaluate this end point, the consistency between these findings and the increased risk of bleeding among patients aged 75 years or more in the enoxaparin group of the SYNERGY trial suggests that this is likely to be a real effect. ${ }^{6}$

Armstrong and colleagues' study ${ }^{1}$ raises several questions, the most important of which may be the optimal anticoagulant and dose to use in combination with fibrinolytics in the treatment of STEMI, and whether that dose can be optimized according to patient age to maximize safety. The ExTRACTTIMI (Enoxaparin and Thrombolysis Reperfusion for Acute Myocardial Infarction-Thrombolysis in Myocardial Infarction) 25 study is comparing enoxaparin with unfractionated heparin in 2I 000 patients whose STEMI was treated with thrombolytic therapy. After years of smaller trials suggesting (but not proving) an efficacy benefit of enoxaparin over unfractionated heparin, the ExTRACT trial will finally provide more definitive results. It will provide important information on the efficacy and safety of reduced doses of enoxaparin in elderly patients. The examination of data combined from the ASSENT- 3 and ASSENT- 3 PLUS trials has been intriguing and useful for hypothesis generation, but real answers should come from the ExTRACT study. ${ }^{8}$

The authors are with the Division of Cardiovascular Medicine, Gill Heart Institute, University of Kentucky, Lexington, Ky.

Competing interests: None declared by Alison Bailey. Steven Steinhubl has received honoraria for advisory-board participation and consultancy fees from Sanofi-Aventis, The Medicines Company, Eli Lilly and AstraZeneca.

Contributors: Both authors conceived the editorial and were responsible for data analysis and interpretation. Steven Steinhubl drafted the commentary. Both authors participated in critical revision of the manuscript and approved the version to be published.

\section{REFERENCES}

I. Armstrong PW, Chang WC, Wallentin L, et al. Efficacy and safety of unfractionated heparin versus enoxaparin: a pooled analysis of ASSENT-3 and -3 PLUS data. CMAJ 2006;174(I0):I42I-6.

2. Assessment of the Safety and Efficacy of a New Thrombolytic Regimen (ASSENT) 3 Investigators. Efficacy and safety of tenecteplase in combination with enoxaparin, abciximab, or unfractionated heparin: the ASSENT-3 randomised trial in acute myocardial infarction. Lancet 2001;358:605-13.

3. Wallentin L, Goldstein P, Armstrong PW, et al. Efficacy and safety of tenecteplase in combination with the low-molecular-weight heparin enoxaparin or unfractionated heparin in the prehospital setting: the Assessment of the Safety and Efficacy of a New Thrombolytic Regimen (ASSENT)-3 PLUS randomized trial in acute myocardial infarction. Circulation 2003;108:135-42.

4. Eikelboom JW, Quinlan DJ, Mehta SR, et al. Unfractionated and low-molecularweight heparin as adjuncts to thrombolysis in aspirin-treated patients with STelevation acute myocardial infarction: a meta-analysis of the randomized trials. Circulation 2005;112:3855-67.

5. Sabatine MS, Morrow DA, Montalescot G, et al. Angiographic and clinical outcomes in patients receiving low-molecular-weight heparin versus unfractionated heparin in ST-elevation myocardial infarction treated with fibrinolytics in the CLARITY-TIMI 28 trial. Circulation 2005;II2:3846-54.

6. Ferguson JJ, Califf RM, Antman EM, et al. Enoxaparin vs unfractionated heparin in high-risk patients with non-ST-segment elevation acute coronary syndromes managed with an intended early invasive strategy: primary results of the SYNERGY randomized trial. JAMA 2004;292:45-54.

7. Giugliano RP, McCabe CH, Antman EM, et al; Thrombolysis In Myocardial Infarction (TIMI) Investigators. Lower-dose heparin with fibrinolysis is associated with lower rates of intracranial hemorrhage. Am Heart J 200I;I4I:742-50.

8. Antman EM, Morrow DA, McCabe CH, et al; for the ExTRACT-TIMI 25 Investigators. Enoxaparin versus unfractionated heparin with fibrinolysis for ST-elevation myocardial infarction. N Engl J Med 2006;354(I4):I477-88.

Correspondence to: Dr. Steven R. Steinhubl, goo S. Limestone St., 326 Charles T. Wethington Building, Lexington $K Y$ 40536-0200, USA; fax 859 323-6475; steinhubl@uky.edu 